\title{
Solution Methods for the Periodic Petrol Station Replenishment Problem
}

\author{
C Triki \\ Mechanical and Industrial Engineering Department, College of Engineering, Sultan Qaboos University, PO Box 33, PC \\ 123, Al-Khoud, Muscat - Sultanate of Oman
}

Received 19 June 2012; accepted 20 February 2013

\begin{abstract}
Abstract: In this paper we introduce the Periodic Petrol Station Replenishment Problem (PPSRP) over a T-day planning horizon and describe four heuristic methods for its solution. Even though all the proposed heuristics belong to the common partitioning-then-routing paradigm, they differ in assigning the stations to each day of the horizon. The resulting daily routing problems are then solved exactly until achieving optimalization. Moreover, an improvement procedure is also developed with the aim of ensuring a better quality solution. Our heuristics are tested and compared in two real-life cases, and our computational results show encouraging improvements with respect to a human planning solution
\end{abstract}

Keywords: Petrol delivery, Periodic constraints, Vehicle routing problem

$$
\begin{aligned}
& \text { طرق حل مشكلة إمدادات محطات البنزين بطريقة دورية } \\
& \text { شافي التريكي }
\end{aligned}
$$

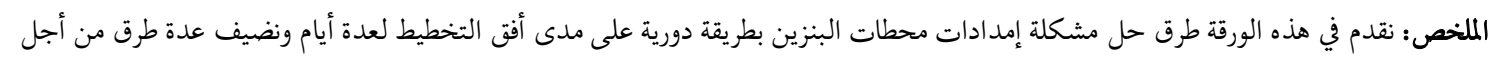

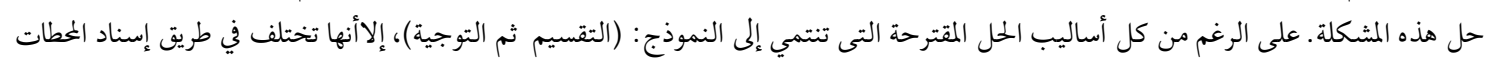

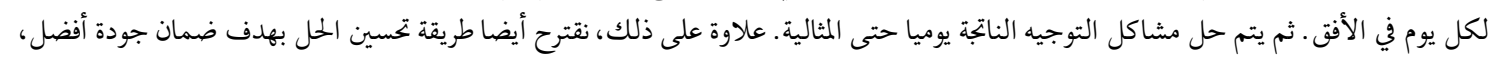

$$
\begin{aligned}
& \text { ويتم اختبار طرق الحل على مثيلين من مشكلة مستخرجة من واقع الحياة. يظهر البحث نتائج مشجعة بالمقارنة مع الحل المعتمد من قبل الشركة المعنية. } \\
& \text { المفردات المفتاحية: تسليم البنزين، توزيع دوري، مشكلة توجيه السيارة. }
\end{aligned}
$$

\section{Introduction}

The problem of planning petrol delivery to distribution stations is well-recognized in the operations research literature under the subject name petrol station replenishment problem (PSRP). It consists of simultaneously making several decisions, including determining the minimum number of trucks required, assigning the stations to the available trucks, defining a feasible route for each truck, settling whether drivers' work overtime is required, etc. The objective to be achieved is usually defined as the minimization of the traveled distance by the trucks to serve all of the distribution stations.
Most of the studies presented in the literature solve this problem over a time period of one single day, ignoring the possibility that solving the PSRP over a $T$ day planning horizon may yield delivery savings for the company (Cornillier et al. 2012). However, most of the PSRPs are characterized by the fact that the stations should not be served at each day of the $T$-day planning horizon but rather through a specified number of times. This would introduce a further characterization of the PSRP; namely, the periodic nature of the application. Specifically, each station (i) must be

*Corresponding author's e-mail: chefi@squ.edu.om 
served $\left(r_{i}\right)$ on certain days within the time horizon, and these service days are assigned to $i$ by selecting one of the feasible combinations of $r_{i}$ service days with the objective of minimizing the total distance traveled by the delivery trucks.

In this paper, we define a new variant of the above described problem that we will denote as the periodic petrol station replenishment problem (PPSRP). We propose a novel three-phase heuristic method for its solution. Our approaches consist of first assigning the service combinations by solving different integer programming models, then defining the routing for each day and, finally, using a local improvement technique to further reduce the delivery distance. A real-life application was chosen for testing and assessing the performance of our solution methods. For some of the proposed heuristics, satisfactory results were obtained with respect to the solution currently adopted by the company.

The paper is organized as follows: the next section, section 2, will be devoted to surveying the related works proposed in the literature and highlighting the original contribution of this paper. Section 3 will formally define the PPSRP and describe the periodicity constraints to be implemented. Section 4 will be dedicated to the development of our heuristic approaches to solve the PPSRP. The computational performance of the heuristics will be discussed in section 5 and, finally, some concluding remarks and future developments will be drawn in section 6 .

\section{Literature Review}

The problem introduced in this paper finds its root in two different classes of distribution problems that are broadly studied in the literature; namely, the PSRP and the periodic vehicle routing problem (VRP). From one perspective, the PPSRP is a natural extension of the PSRP as it prolongs the planning horizon to cover a $T$-day period. The 1-day variant of the problem has been subjected to extensive research activity, and several mathematical models and numerical methods have been proposed for its solution. A limited list of these works includes a collection of papers by (Cornillier et al. (2008a, 2008b, 2009, 2012) and the contributions of (Brown and Graves 1981; Brown et al. 1987; Ben Abdelaziz et al. 2002; Rizzoli et al. 2003; Ng et al. 2008; Surjandari et al. 2011 and Boctor et al. 2012). Only a few papers have considered the multi-period aspect in the context of PSRP, but none of them included explicitly the periodic nature of the problem.
Taqa Allah et al. (2000) specifically have extended the planning horizon of the PSRP to the multi-period case and have proposed several heuristic methods for the solution of the so-called MPSRP. In their paper, they examined a single depot and an unlimited homogeneous truck fleet.

Malepart et al. (2003) also tackled the multi-visit petrol station replenishment problem (MPSRP) in a real-life context and included a case in which the distribution company would decide for some stations the quantity to be delivered at each visit.

Cornillier et al. (2008b) penned the most recent work, which considered a multi-period horizon and proposed a heuristic that maximizes the profit, which is calculated as the difference between the delivery revenue and the sum of regular and overtime drivers' costs.

Thus, to the best of our knowledge this paper represents a first tentative step in tackling a PSRP which is characterized by the periodic service requirements of the petrol stations.

On the other hand, the PPSRP can find its roots in the literature of the periodic VRP that attracted the interest of many researchers over the previous two decades (Gaudioso and Paletta 1992; Chao et al. 1995; Cordeau et al. 1997). The two problems have many similarities, but the main difference between the two consists of the fact that the PPSRP should include additional constraints (for example, those related to the drivers' shifts' lengths).

\section{Problem Description}

The PPSRP is formally defined in terms of graph theory on an undirected connected network $\mathrm{G}=(V$, $A)$. The set of vertices $V=\{1, \ldots, i, \ldots, n\}$ includes all the stations to be served whereas vertex 0 denotes the petrol depot. With $A=\{(i, j): \forall i$ and $j \in V \cup\{0\}\}$, we denote the set of all undirected edges that connect all the vertices of the network. To each edge $(i, j) \in A$ is associated a traversal cost $\left(c_{i j}\right)$ that generally represents its length. An example of a region to be served and its resulting graph are represented in Fig. 1.

The objective of the PPSRP consists of finding a minimum distance routing to serve all the stations when considering an extended planning horizon of $T$ days.

Within this $T$-day horizon, each station $i$ must be visited $r_{i}$ times, with at most one visit per day. These visits are assigned to $i$ by selecting one out of a given set of feasible combinations of $r_{i}$ visit days. Several 

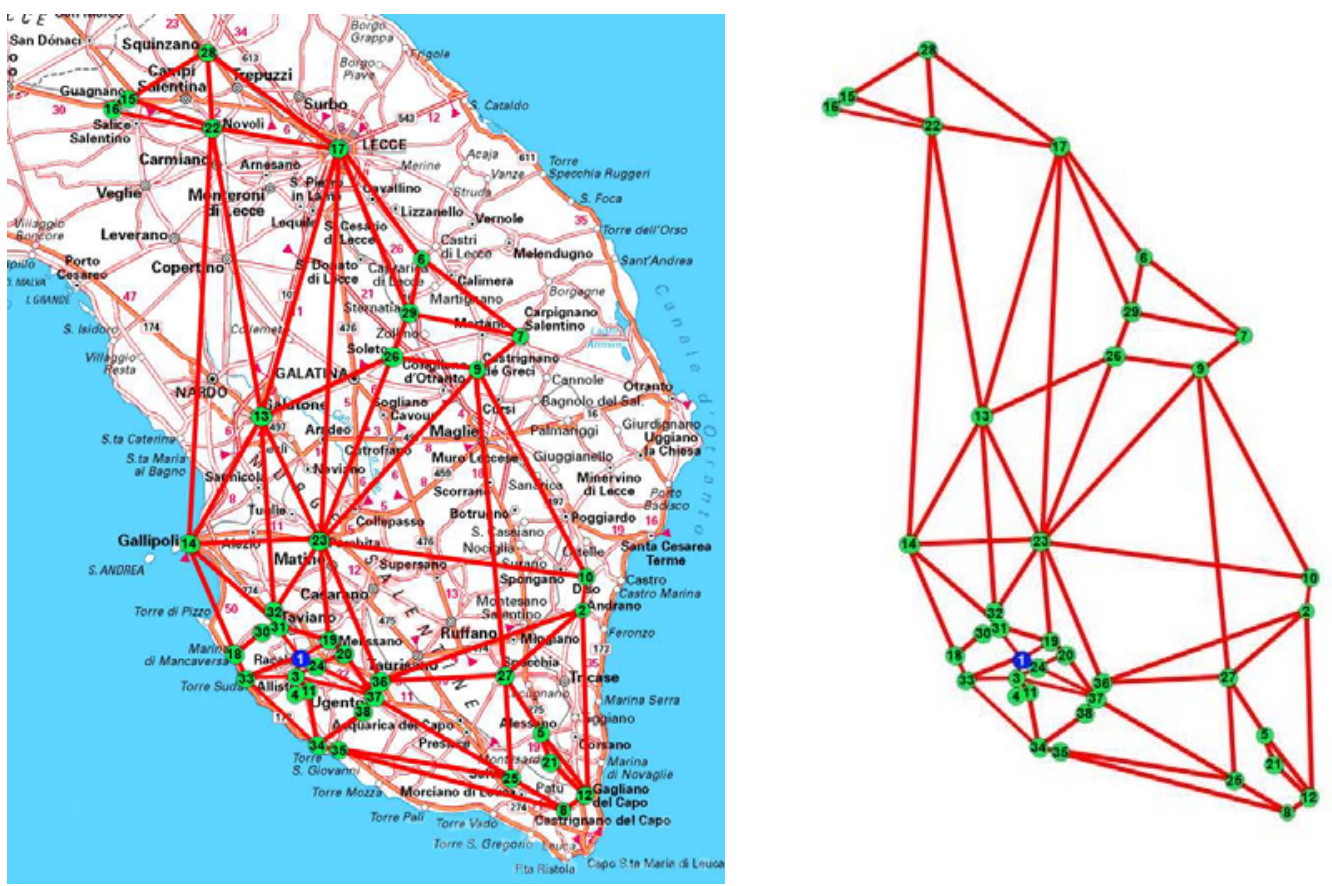

Figure 1. An example of a region to be served (left) and its corresponding connected network (right). The stations correspond to the nodes of the graph and the roads are the undirected edges

mathematical representations of the periodicity aspect within the routing problems have been discussed (Paletta and Triki 2004). In this paper we will suppose that the set $C_{i}$ for each city $i \in V$ is defined on the basis of the periodic sequence of combinations. Consequently, each station specifies the number of service visits $\left(r_{i}\right)$ during the $T$-day planning horizon, and it must be visited every $T / r_{i}$ days. An example of feasible combinations for $T=6$ is reported in Table 1.

Table 1. Periodic sequence combinations for $T=6$

\begin{tabular}{cc}
\hline $\begin{array}{l}\text { Frequency } \\
\text { of service } \boldsymbol{r}_{i}\end{array}$ & $\begin{array}{l}\text { Feasible sequence of } \\
\text { combinations } C_{i}\end{array}$ \\
\hline 1 & $C_{i}=\{(1),(2),(3),(4),(5),(6)\}$ \\
2 & $C_{i}=\{(1,4),(2,5),(3,6)\}$ \\
3 & $C_{i}=\{(1,3,5),(2,4,6)\}$ \\
6 & $C_{i}=\{(1,2,3,4,5,6)\}$ \\
\hline
\end{tabular}

The resulting network model can be expressed in terms of combinatorial optimization having two sets of decision variables. The first set defines the routing solution and the corresponding truck assignment, (ie. it will give clear insight on which truck will serve which stations in which day in order to satisfy all the demand). The second set of variables will choose for each station the best periodicity combination that will minimize the overall delivery distance. The integer model with all its constraints is cumbersome and rep- resents only slight differences with respect to the mathematical model reported (Cornillier et al. 2008b). For this reason and for brevity we avoid reporting the whole model in this paper and we focus in the sequel on the innovative heuristic methods developed for its solution.

\section{Heuristic Algorithms}

Since PPSRP instances are generally characterized by a high number of variables and constraints due mainly to the extended time horizon and also to the large number of stations to be served, we have chosen to solve the problem heuristically. We propose here four different heuristics all based on the idea of splitting the task of assigning the periodicity combinations to the stations from that of performing the routing rather than dealing with both the tasks simultaneously (Fig. 2). The routing task then is carried out by solving the resulting VRPs-one for each day of the planning horizon. Finally, all of the heuristics are concluded by running an improvement procedure that is common to all four approaches.

\subsection{Combination Assignment Procedure}

The procedure of assigning feasible combinations to the stations is carried out by solving exactly, using any general purpose software, one of the integer programming models proposed below. The notation to be used through this section is the following: 


$$
\begin{array}{ll}
T & \text { days of the planning horizon } \\
n & \text { number of stations } \\
d_{i t} & \begin{array}{l}
\text { demand of petrol of station } i \in V \text { for day } t \\
\text { (ie. weekly demand of } i \text { divided by } r_{i} \text { ) }
\end{array} \\
C_{i} & \begin{array}{l}
\text { feasible periodicity co mbinations for } \\
\text { station } i \in V
\end{array} \\
a_{r t} & \begin{array}{l}
\text { input constant that equals } 1 \text { if day } t \text { belongs } \\
\text { to combination } r, \text { and } 0 \text { otherwise }
\end{array} \\
N V & \text { number of available vehicles } \\
Q_{v} & \text { capacity of vehicle } v=1, \ldots, N V \\
c_{i j} & \text { distance from station } i \text { and station } j \text { with } \\
& i, j \in V \cup\{0\}
\end{array}
$$

\subsubsection{Heuristic 1: Minimum Daily Demand}

The idea here is to minimize the total demand to be served on each single day of the planning horizon. In this way, the workload will be as balanced as possible and, consequently, the number of vehicles will be minimized on a daily basis. While this procedure has the advantage of being easy to understand and implement, it has the disadvantage of not taking into account the geographical position of the stations. The proposed integer program has $y_{i r}$ as the decision variables that define the periodicity combination to be assigned to each station, as follows:

$y_{i r}$ is 1 if combination $r$ is assigned to station $i, 0$ otherwise.

Moreover, by denoting by $D_{t}$ the total quantity of petrol to be served on day $t$, the mathematical model can be expressed as:

$$
\operatorname{Min} \underset{t=1, \ldots T}{\operatorname{Max}} D_{t}
$$

Subject to

$$
\begin{aligned}
& \sum_{r \in C_{i}} y_{i r}=1 \quad i=1, \ldots, n \\
& \sum_{i=1}^{n} \sum_{r \in C_{i}} a_{r t} d_{i t} y_{i r} \leq D_{t} \quad t=1, \ldots, T \\
& y_{i r} \in\{0,1\} \quad r \in C_{i}, i=1, \ldots, n
\end{aligned}
$$

The objective function (1) minimizes the highest quantity of petrol to be served over all the days of the planning horizon. It is expressed here as a min-max objective function but it can be easily transformed to a standard minimization problem by using conventional operations research techniques. Constraints (2) force the model to choose only one periodic combination among all the feasible combinations. Constraints (3) restrict the daily quantity $\left(D_{t}\right)$ to be delivered to match at least the total demand to be served on day $t$. Finally, constraints (4) impose the domain of the decision variables.

\subsubsection{Heuristic 2: Minimum Daily Stations}

The idea of this heuristic is to minimize the per day total number of stations to be served. Even in this case, the attempt aimed at balancing the workload was expressed in terms of the number of stations and, consequently, at minimizing the number of vehicles to be used on a daily basis. This heuristics shares the same advantage as its predecessor of being easy to understand and implement and, moreover, ensures a balanced workload for the trucks over the $T$ days of the horizon. Both the heuristics also share the same disadvantage of ignoring the geographical position of the stations.

By using the same binary variables $y_{i r}$ as defined previously and by introducing a new variable $W_{n t}$ defining the number of stations to be visited during day $t$, the mathematical model is expressed as:

$$
\begin{aligned}
& \underset{t=1, \ldots T}{\operatorname{Min} \underset{t}{\operatorname{Max}} W_{n t}} \\
& \text { Subject to : } \\
& \sum_{r \in C_{i}}^{n} y_{i r}=1 \quad i=1, \ldots, n \\
& \sum_{i=1}^{n} \sum_{r \in C_{i}} a_{r t} y_{i r}=W_{n t} \quad t=1, \ldots, T \\
& y_{i r} \in\{0,1\} \quad r \in C_{i}, i=1, \ldots, n \\
& W_{n t} \text { Integer } \quad t=1, \ldots, T
\end{aligned}
$$

The objective function (5) makes use of the new variable $W_{n t}$ in order to minimize the highest number of stations to be served over all the days of the planning horizon. Constraints (6) assigns as before only one periodic combination to each station. Constraint (7) imposes logic relations between the new variable $W_{n t}$ and the assignment variables $y_{i r}$ on a daily basis, and the domain of the decision variables is defined through constraints (8) and (9).

\subsubsection{Heuristic 3: Depot Minimum Distance}

The aim of this heuristic is to solve a relaxation of a PVRP-like process in order to minimize the total distance between the depot (node 0 ) and all the stations to be served. Even though the resulting model is a simplified version of the PVRP, it will maintain some of the complexity but on the other hand it has the advantage of making use of more information since it takes into consideration the geographical position of the stations with respect to the depot.

The integer model has two sets of variables. Besides, the binary variables $y_{i r}$ as previously used we need to introduce also the following binary variables:

$x_{0 i t}$ is 1 if station $i$ is assigned to the depot (node 0) on day $t$, and 0 otherwise. 


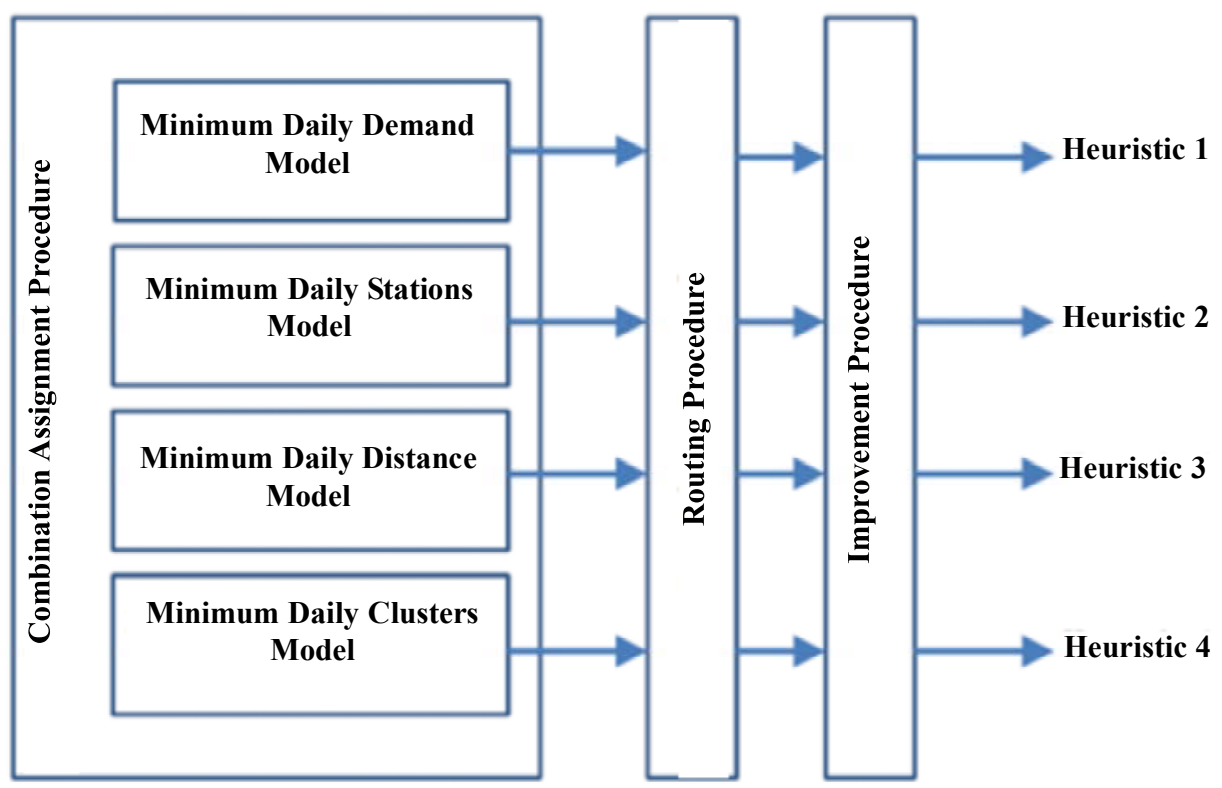

Figure 2. The four heuristic solution approaches

The optimization model can be summarized as follows:

$$
\text { Minimize } \sum_{\mathrm{i}=1}^{\mathrm{n}} \sum_{\mathrm{t}=1}^{\mathrm{T}} \mathrm{c}_{0 \mathrm{i}} \mathrm{x}_{0 \mathrm{it}}
$$

Subject to:

$$
\begin{aligned}
& \sum_{r \in C_{i}} y_{i r}=1 \quad i=1, \ldots, n \\
& x_{0 i t}=\sum_{r \in C_{i}} a_{r t} y_{i r} \quad i=1, \ldots, n, t=1, \ldots, T \\
& \sum_{i=1}^{n} d_{i t} x_{0 i t} \leq \sum_{v=1}^{N V} Q_{v} \quad t=1, \ldots, T \\
& x_{0 i t} \in\{0,1\} \quad i=1, \ldots, n, t=1, \ldots, T \\
& y_{i r} \in\{0,1\} \quad i=1, \ldots, n, r \in C_{i}
\end{aligned}
$$

The objective function (10) minimizes the total distance of all the stations with respect to the depot over the days of the planning horizon. Constraints (11) has the same role as in the previous models, whereas constraint (12) imposes logic relations between the two sets of variables $x_{0 i t}$ and $y_{i r}$. The inequalities of (13) are capacity constraints that ensure, for each day $t$, the respect of the trucks' capacity and, finally, the integrity of the decision variables that are defined by constraints (14) and (15). It should be noted that this model is similar to that in (1) through (4). The main difference between the two problems lies in the objective function (1), which becomes (10).

\subsubsection{Heuristic 4: Minimum Distance Clusters}

This heuristic is an extension of the previous one, allowing the minimization of the total distance of the stations with respect to virtual centers, with one for each day of the horizon, rather than considering only the depot. This clustering strategy allows better use of the information about the distances between stations and, hopefully, generates more compact sets of stations from a geographical viewpoint. The obvious disadvantage is the complexity of the resulting model involving a large number of binary variables.

For simplicity, the virtual centers are chosen here among a set of specific positions that coincide with some of the stations, so that the topology of the network remains unaffected. The integer model makes use of the variables $y_{i r}$ and, moreover, introduces the following two sets of binary variables:
$x_{i j t} \quad$ is 1 if station $\mathrm{i}$ is assigned to the virtual cen- tre $j$ in day $t$, and 0 otherwise
$z_{j t} \quad$ is 1 if virtual centre $j$ is assigned to day $t$, and 0 otherwise

The integer model corresponding to heuristic 4 is as follows:

Minimize $\sum_{i=1}^{n} \sum_{j=1}^{T} \sum_{t=1}^{T} c_{i j} x_{i j t}$

Subject to:

$$
\sum_{\mathrm{r} \in \mathrm{C}_{\mathrm{i}}} \mathrm{y}_{\mathrm{ir}}=1 \quad \mathrm{i}=1, \ldots, \mathrm{n}
$$

$\sum_{j=1}^{T} x_{i j t}=\sum_{r \in C_{i}} a_{r t} y_{i r} \quad i=1, \ldots, t=1, \ldots, T$

$\sum_{j=1}^{T} z_{j t}=1 \quad t=1, \ldots, T$ 


$$
\begin{aligned}
& \sum_{i=1}^{n} d_{i t} x_{i j t} \leq\left(\sum_{v=1}^{N V} Q_{V}\right) z_{j t} t=1, \ldots, T, j=1, \ldots, T \\
& x_{i j t} \in\{0,1\} \quad i=1, \ldots, n, t=1, \ldots, T, j=1, \ldots, T \\
& y_{\text {ir }} \in\{0,1\} \quad i=1, \ldots, r \in C_{i} \\
& z_{j t} \in\{0,1\} \quad t=1, \ldots, T, j=1, \ldots, T
\end{aligned}
$$

Similar to the previous heuristic, the objective function (16) minimizes the total distance of all the stations with respect to the $T$ virtual centres (one for each day of the planning horizon). All the other constraints have the same meaning and role as in the previous heuristic except for some slight adjustments due to presence of $T$ virtual centres and the introduction of the new set of variables $z_{j t}$. We should mention that the notation may be further simplified in the above model by merging the indexes $\mathrm{j}$ and $\mathrm{t}$ since they represent the same set of horizon days and have a one-to-one relationship but we preferred, for the sake of clarity, to distinguish between the temporal index $t$ and the spatial index $j$.

Finally, we should note that the last two heuristics seem to be more challenging computationally since they include the geographical aspect of the problem and, thus, involve additional decision variables with respect to the former two methods. Possible higher quality solutions to be yielded by the latter heuristics may be paid by longer computational time when solving large-scale problems. This time-quality trade off may be very useful when solving several instances with different sizes.

\subsection{Solving the VRPs: Routing Procedure}

Once the hard task of assigning the stations to the days of the planning horizon while keeping in mind the periodicity constraints on each station has been solved, we need to define the routes for each day. The routing procedure takes as input the sets of stations corresponding to each day (ie. the output of any of the models of subsection 4.1) and generates a set of routes for the available trucks. This requires nothing more than solving independently one $T$ standard VRP for each day of the horizon. Since each resulting VRP is relatively small with respect to the original PPSRP, we decided to solve these $T$ problems exactly by using a standard VRP model and a general purpose optimization software package.

\subsection{Getting a Better Solution: Improvement Procedure}

The final step of our approach consisted of trying to improve the quality of the solution by further minimizing the overall distance through the application of a local search technique. Theoretically, room for its improvement should be available since the assignment of the stations to the $T$ days has been done heuristically without any guarantee to get the best feasible solution. Starting from a set of routes assigned to the available trucks for every day of the planning horizon, the technique employed here is based on switching any two stations assigned to two different service days and checking if any improvement is taking place. While checking the improvement in the total distance at each switching station, we should check also the feasibility of the solution expressed in terms of service periodicity of each station and the capacity of the corresponding trucks. This procedure is easy to understand but requires considerable effort for its implementation, since no standard software is available to achieve this task.

\section{Computational Results}

This section is dedicated to the application of our heuristic methods to solving a real-life test problem, and to analyzing and comparing their performances. All the models introduced above were implemented using the LINGO commercial package, Version 8.0 (LINDO Systems, Inc., Chicago, Ill., USA) as modeling language that has been interfaced with the state-ofthe-art solver CPLEX, Version 12.1 (IBM ILOG, Gentilly, France) for the solution of the resulting integer programs. On the other hand, the improvement procedure required specific programming skills and, thus, the language JAVA has been chosen for its implementation. A computer with an Intel Core 2 Duo Processor $2.53 \mathrm{GHz}$ and $4 \mathrm{~GB}$ of RAM was used to run all the resulting codes.

\subsection{Real-life Test Problem}

To our knowledge, there are no standard test problems available as a benchmark for the PPSRP, since we believe it is the first time this problem is being tackled. For this reason, we will compare the quality of our heuristics' solutions from one side with respect to the solution currently being adopted by the delivery company, and from the other with respect to the best feasible solution that can be reached by the exact solver.

This case study consisted of a regional company located in the south of Italy having a central petrol depot from which a heterogeneous fleet of trucks starts daily trips to serve 38 petrol stations located in different places throughout the regional territory. The planning horizon for this problem is a week consisting of 6 work days $(T=6)$. The weekly demand for petrol varies from one station to another requiring in some cases only one delivery visit; however, most depots require a multi-visit service fractioned into 2 or 3 deliveries per week. The available trucks have differ- 


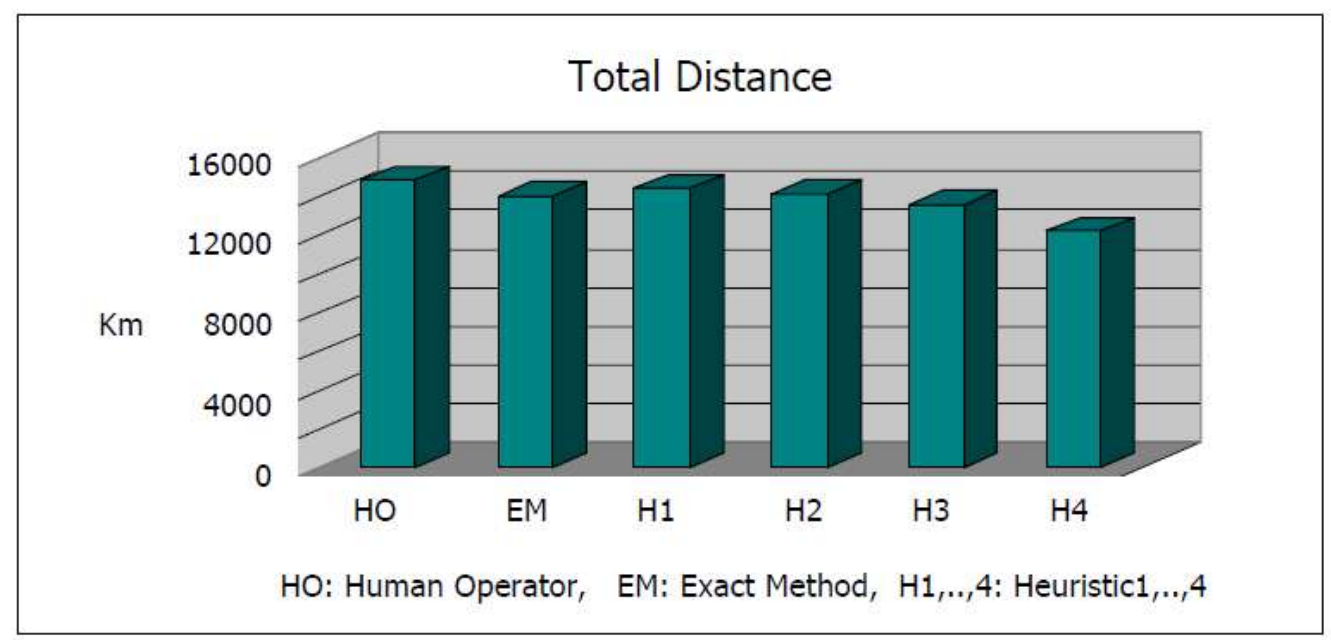

Figure 3. Comparison between our solutions and the company's solution

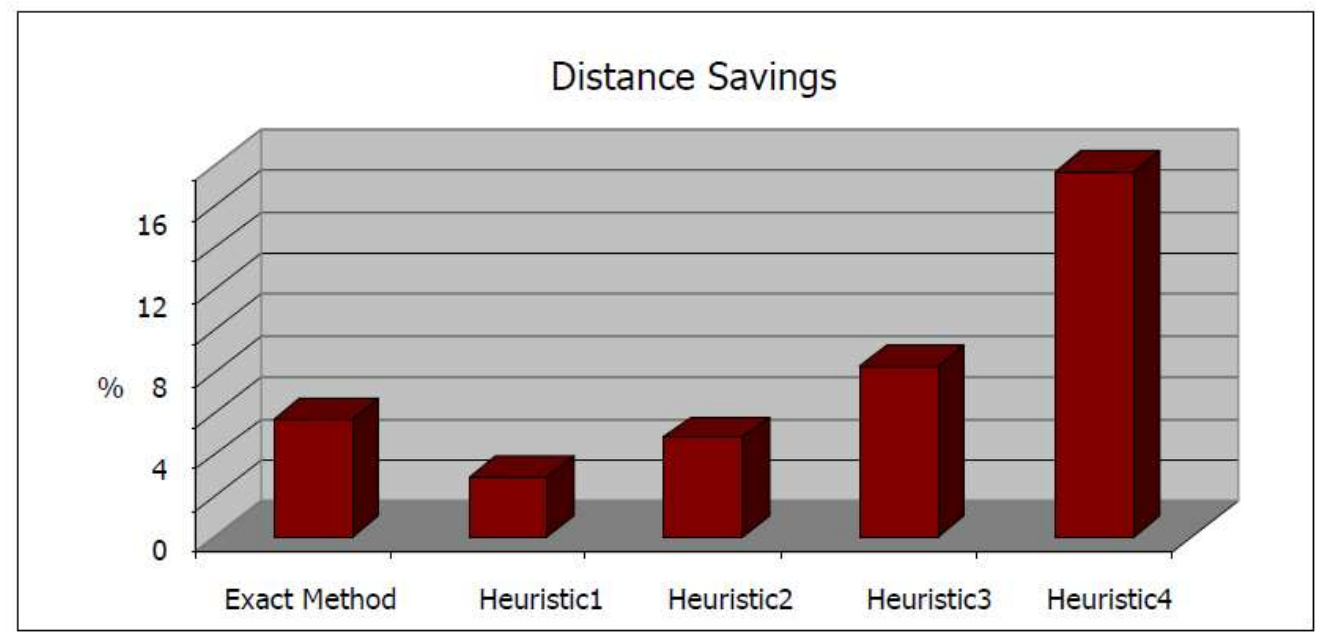

Figure 4. Savings obtained with respect to company's solution

ent capacities, allowing the model to choose for each route the truck that best fits the model's constraints.

Concerning the drivers, generally speaking the company has enough drivers available during each weekday except for the sixth working day (ie. the first weekend day) in which the number of drivers is limited because of the unavailability of most of them to work. This limitation has been considered in our models by dropping, whenever is possible, the sequence combinations that involve day 6 . For example, when $r_{i}=2$, we have considered only the set $C_{i}=\{(1,4$. $.2,5)\}$ as feasible combinations and we disregarded the combination $(3,6)$ in order to take into account the drivers' unavailability. We kept, however, both the sequences $C_{i}=\{(1,3,5 . .2,4,6)\}$ for the stations requiring 3 visits per week.

The integrated PPSRP model corresponding to our application resulted in a large-scale problem having 84,740 constraints and 84,701 variables. The state-ofthe-art CPLEX software package was not able to yield an exact solution for the problem but generated at least a feasible upper bound solution of 13,975 km/week. Indeed, after more than $30 \mathrm{CPU}$ hours and a huge number of iterations, the software presented a faulty memory error due to the large number of sub problems generated during the branch-and-bound method.

We also collected data for a different aspect of the test problem in order to ascertain information about the performance of our heuristic procedures. The different aspect refers to another working week with different demands for petrol and also different frequencies of periodicity.

\subsection{Results and Discussions}

We started by assessing the solution quality of each approach (including the exact method) before and after applying the improvement procedure and the corresponding CPU time. The results collected in Table 2, show, from one side, the superiority of heuristic 4 , both in its solution quality and CPU time, with respect to the other heuristics and also with respect to the feasible upper bound obtained by CPLEX. From the 
Table 2. Solution costs (in terms of distance) and CPU times

\begin{tabular}{lccc}
\hline Approach & $\begin{array}{c}\text { Initial So lution (Km) } \\
\text { Before Improvement }\end{array}$ & $\begin{array}{c}\text { Final Solution (Km) } \\
\text { After Improvement }\end{array}$ & $\begin{array}{c}\text { Total CPU Time } \\
\text { (sec.) }\end{array}$ \\
\hline Exact Method & 13975 & 13360 & 109628 \\
Heuristic 1 & 14382 & 14281 & 1021 \\
Heuristic 2 & 13744 & 13036 & 3264 \\
Heuristic 3 & 13587 & 13340 & 2126 \\
Heuristic 4 & 12193 & 12193 & 591 \\
\hline
\end{tabular}

Table 3. Comparison between our solutions and the company's solution

\begin{tabular}{lccc}
\hline Approach & Distance $\mathbf{( K m )}$ & Savings $\mathbf{( k m )}$ & Savings (\%) \\
\hline Human Operator & 14820 & ------ & ----- \\
Exact Method & 13360 & 1469 & 9.9 \\
Heuristic 1 & 14281 & 539 & 3.6 \\
Heuristic 2 & 13036 & 1784 & 12.0 \\
Heuristic 3 & 13340 & 1480 & 10.0 \\
Heuristic 4 & 12193 & 2627 & 17.7 \\
\hline
\end{tabular}

Table 4. Comparison of the solutions for different test data

\begin{tabular}{lccc}
\hline Approach & $\begin{array}{c}\text { Initial Solution (Km) } \\
\text { Before Improvement }\end{array}$ & $\begin{array}{c}\text { Final solution (Km) } \\
\text { After Improvement }\end{array}$ & $\begin{array}{c}\text { Savings (\%): Final vs. } \\
\text { Company's solutions }\end{array}$ \\
\hline Human Operator & 14770 & ------- & ------ \\
Heuristic 1 & 15053 & 14814 & ----- \\
Heuristic 2 & 14197 & 13987 & 5.3 \\
Heuristic 3 & 12848 & 12828 & 13.1 \\
Heuristic 4 & 12819 & 12819 & 13.2 \\
\hline
\end{tabular}

other side, the positive effect of the improvement procedure on all the methods, except heuristic 4 , is clear. This means that the solution generated by heuristic 4 is probably close enough to the optimal solution so that no margin was left for improvement.

The second set of experiments deals with the assessment of our approaches with respect to the solution currently adopted by the company. Such a solution has not been obtained through the use of any decision support tool but just as the result of a long experience of the company's human operators in managing not only the loading/unloading operations but also the transportation phase. For the data corresponding to the week we took into consideration, the actual distance run by the trucks for serving all 38 stations was 14,820 kms. It is evident from Table 3 that all our approaches improve the human operator solution and, particularly, heuristic 4 will reach, after improvement, a relative distance saving of $17.7 \%$ with respect to the compa- ny's decisions. These same results are also depicted in Figs. 3 and 4.

Finally, for the sake of completeness, we tested our heuristic procedures on a different set of data for the same distribution network. This kind of sensitivity analysis experiment has the aim of checking the robustness of the obtained solutions, and specially verifying the conclusions related to heuristic 4 . This new set of data has been purposely collected for another week belonging to a different season and with a different demand pattern with respect to the previous data. The two sets differ only by the amount of petrol to be served to each station and, consequently, by their frequency of service.

The results reported in Table 4 show that these new results substantially confirm the trend observed in the previous test (except for heuristic 1). More specifically, heuristic 4 once again outperforms all the other approaches. Moreover, even in this case the improve- 
ment procedure has no effect on the initial solution obtained, confirming our intuitive conclusion that heuristic 4 generates a nearly optimal solution.

From another point of view, the results reported in the final column of Table 4 confirm the improvement that three of our heuristics can achieve with respect to the solution adopted by the company for that week.

\section{Conclusions}

In this paper, we presented and discussed the PPSRP. We proposed four heuristic approaches based on three phases each: the assignment of periodicity combinations to each station, the construction of trucks' delivery routes, and, finally, the routes' improvement phase. The performance of the proposed heuristics was tested on two different instances of a real-life periodic problem. Both instances showed the superiority of heuristic 4 in relation to the others, reaching an average improvement of more than $15 \%$ in terms of traveled distance, with respect to the solutions actually adopted by the company while keeping the execution time within reasonable limits. Future developments in this direction should consist of solving another more challenging real-life problem on a national scale for a petrol distribution company operating in Oman. Besides the difficulties that will be introduced by the problem's size, the new application also might be characterized by further complexities such as uncertainty related to the drivers' availability and regulatory restrictions on the citizenship of the drivers to accomplish some routes.

\section{Acknowledgment}

Thanks are due to the anonymous referees for their valuable comments, which improved the quality of the paper.

\section{References}

Ben Abdelaziz F, Roucairol C, Bacha C (2002), Deliveries of liquid fuels to SNDP gas stations using vehicles with multiple compartments. In: Systems Managment and Cybernetics IEEE International Conference. Hammamet, Tunisia.

Boctor F, Renaud J, Cornillier F (2011), Trip packing in petrol stations replenishment. Omega 39:86-98.

Brown GG, Ellis CJ, Graves GW, Ronen D (1987), Real-time wide area dispatch of mobil tank trucks. Interfaces 17:107-120.

Brown GG, Graves GW (1981), Real-time dispatch of petroleum tank trucks. Management Science 27:19-32.

Chao IM, Golden BL, Wasil E (1995), An improved heuristic for the period vehicle routing problem.
Networks 26:25-44.

Christofides N, Beasley JE (1984), The period routing problem. Networks 14:237-256.

Cordeau JF, Gendreau M, Laporte G (1997), A tabu search heuristic for periodic and multi-depot vehicle routing problems. Networks 30:105-119.

Cornillier F, Boctor F, Laporte G, Renaud J (2008a), An exact algorithm for the petrol station replenishment problem. Journal of the Operational Research Society 59:607-615.

Cornillier F, Boctor F, Laporte G, Renaud J (2008b), A heuristic for the multi-period petrol station replenishment problem. European Journal of Operational Research 191:295-305.

Cornillier F, Laporte G, Boctor F, Renaud J (2009), The petrol station replenishment problem with time windows. Computers and Operations Research 36:919-935.

Cornillier F, Boctor F, Renaud J (2012), Heuristics for the multi-depot petrol station replenishment problem with time windows. European Journal of Operational Research 220:361-369.

Gaudioso M, Paletta G (1992), A heuristic for the periodic vehicle routing problem. Transportation Science 26:86-92.

IBM ILOG Cplex Optimizer. (2011), Available at: 01.ibm.com/software/integration/optimization/cpl ex-optimizer/. Accessed May 2012.

Lindo Systems Inc. (2006), Optimization Modeling with Lingo. Available at: www.lindo.com. Accessed May 2012.

Malépart V, Boctor F, Renaud J, Labilois S (2003), Nouvelles approches pour l'approvisionnement des stations d'essence. Revue Française de Gestion Industrielle 22:15-31.

Ng WL, Leung SH, Lam JP, Pan SW (2008), Petrol delivery tanker assignment and routing: a case study in Hong Kong. Journal of the Operational Research Society 59:1191-1200.

Paletta G, Triki C (2004), Solving the asymmetric traveling salesman problem with periodic constraints. Networks 44:31-37.

Rizzoli A, Casagrande N, Donati A, Gambardella L, Lepori D, Montemanni R, Pina P, Zaffalon M (2003), Planning and optimisation of vehicle routes for fuel oil distribution. In: MODSIM International Congress on Modelling and Simulation. Townsville, Australia.

Surjandari I, Rachman A, Dianawati F, Wibowo RP (2011), Petrol delivery assignment with multiproduct, multi-depot, split deliveries and time windows. International Journal of Modeling and Optimisation 1:375-379.

Taqa Allah D, Renaud J, Boctor FF (2000), Le problème d'approvisionnement des stations d'essence. APII-JESA Journal Européen des Systèmes Automatisés 34:11-33. 